\title{
Optimum Time Resource Allocation for TDMA-Based Differential Decode-and-Forward Cooperative Systems: A Capacity Perspective
}

\author{
Li Wang and Lajos Hanzo
}

\begin{abstract}
It is widely recognized that differential decode-andforward (DDF) cooperative transmission schemes are capable of achieving a cooperative diversity gain, while circumventing the potentially excessive-complexity and yet inaccurate channel estimation, especially in mobile environments. In this letter, we find the optimum transmit-interval duration for the source and relay, which is commensurate with their adaptive channel-code rate in the context of TDMA-based DDF-aided half-duplex systems for the sake of maximizing the achievable network throughput. We also demonstrate from a pure capacity perspective, in what scenarios the introdution of cooperation improves the achievable throughput.
\end{abstract}

\section{INTRODUCTION}

User-cooperation based transmit diversity techniques [1,2] constitute powerful arrangements of mitigating the deleterious effects of fading by creating a Virtual Antenna Array (VAA) from the single-antenna based shirt-pocket-size wireless devices, hence improving the end-to-end system performance. In order to avoid channel estimation which may impose both an excessive complexity and a high pilot overhead in cooperative systems, especially in mobile environments associated with relatively rapidly fluctuating channel conditions, differentially encoded transmissions combined with non-coherent detection and hence requiring no channel state information (CSI) at the receiver becomes an attractive design alternative, especially in TDMA-based differential modulation assisted cooperative communications [3-6].

However, the recent TDMA-based cooperative system optimization efforts have been mainly focused on power allocation and relay station (RS) selection $[1,6,7]$ based on a fixed and predetermined time resource allocation (TRA) between the source and RS. In this letter, we deduce the optimum TRA policy for the sake of maximizing the DDFaided cooperative system's capacity, by utilizing information theoretical tools, which become useful in the design of nearcapacity coding/decoding schemes conceived for cooperative systems, since the code rate employed by the source and RS is directly related to their allocated transmission duration, and may be adaptively selected according to our proposed TRA scheme. Furthermore, it is important to note that the cooperative diversity gain and the reduced-path-loss-related power gain are achieved by a half-duplex relay at the cost of suffering a significant throughput loss imposed by the relay's transmissions. Hence, in the interest of achieving a high spectral efficiency, we also identify the scenarios, when the introduction of cooperation becomes beneficial from a pure capacity perspective.

The authors are with the school of ECS, University of Southampton, Southampton, SO17 1BJ, UK (e-mail: $\{1 \mathrm{w} 5,1 \mathrm{~h}\} @$ ecs.soton.ac.uk).

Acknowledgements: The finacial support of the EPSRC UK under the auspices of the UK-India centre of Excellence in Wireless Communications and that of the EU Optimix project is gratefully acknowledged.

\section{System Architecture \& Channel Model}

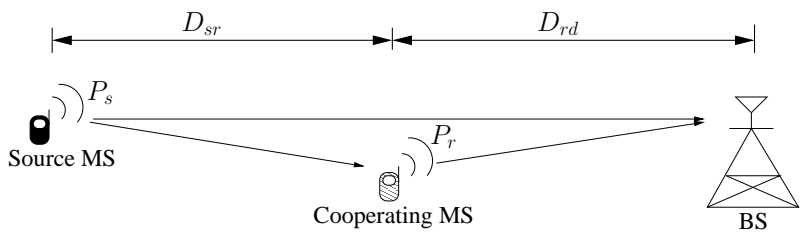

Fig. 1. Single-relay-aided cooperative cellular uplink.

The TDMA-based DDF cooperative cellular uplink (UL) considered is illustrated in Fig. 1, where a single cooperating mobile station (MS) is activated to forward the source MS's signal to the base station (BS). Each MS employs a single antenna, owing to their cost- and size-constraints. In order to avoid CSI estimation, both the source and RS employ conventional differential modulation schemes, such as DQPSK. Since our emphasis is on investigating the TRA, we stipulate the simplifying assumption of equal power allocation and midpoint RS location, as shown in Fig. 1.

In order to provide a good approximation for TDMAbased cooperative systems and to facilitate the non-coherent detection-based system capacity analysis, we consider a timeselective block-fading Rayleigh channel [8], where the fading coefficients exhibit correlation within a fading block according to the normalized Doppler frequency $f_{d}$ induced by the relative movement of the tranceivers, and changes in an i.i.d. manner from block to block.

The signals consecutively received within a fading block size of $T_{b}$ at the RS during the broadcast Phase I, when a total of $L_{s}$ symbols per transmission block are transmitted from the source MS, may be formulated as:

$$
\mathbf{y}_{r}^{I}=\sqrt{P_{s}} \mathbf{S}_{D, s}^{I} \mathbf{h}_{s r}+\mathbf{w}_{r}
$$

where $\mathbf{y}_{r}^{I}, \mathbf{h}_{s r}$, and $\mathbf{w}_{r}$ represent the received signal's column vector, the fading coefficients' column vector obeying a complex-valued Gaussian distribution $\mathcal{C N}\left(0, \sigma_{s r}^{2}\right)$ and the Gaussian noise column vector having a distribution of $\mathcal{C N}\left(0,2 \sigma_{w}^{2}\right)$, respectively. The diagonal matrix $\mathbf{S}_{D, s}^{I}$ may be expressed as $\mathbf{S}_{D, s}^{I}=\operatorname{diag}\left\{\mathbf{s}_{\mathrm{s}}^{\mathrm{I}}\right\}$, where $\mathbf{s}_{s}^{I}$ is the transmitted signal's column vector hosting the $T_{b}$ symbols of a fading block during Phase I. Similarly, the block-wise signal model at the BS during Phases I and II can be expressed respectively as:

$$
\mathbf{y}_{d}^{I}=\sqrt{P_{s}} \mathbf{S}_{D, s}^{I} \mathbf{h}_{s d}+\mathbf{w}_{d}
$$

and

$$
\mathbf{y}_{d}^{I I}=\sqrt{P_{r}} \mathbf{S}_{D, r}^{I I} \mathbf{h}_{r d}+\mathbf{w}_{d} .
$$




\section{The TRA-OptIMIZED NETWORK CAPACITY}

\section{A. Decode-and-Forward Cooperative Network Capacity}

Let us now consider the capacity of the DDF-aided cooperative system of Fig. 1. Based on the general upper and lower bounds on the capacity of half-duplex relay systems presented in [7] and on the fact that in our DDF-aided half-duplex relay system, the source MS remains silent during Phase II, when a total of $L_{r}$ symbols are transmitted by the RS, we may obtain simplified capacity upper and lower bounds for the DDF system of Fig. 1 as:

$$
\begin{aligned}
C_{\text {coop }}\left(\gamma_{e}^{o}, \alpha\right) \leq & \min \left\{\alpha I\left(\mathbf{s}_{s}^{I} ; \mathbf{y}_{d}^{I}, \mathbf{y}_{r}^{I}\right),\right. \\
& \left.\alpha I\left(\mathbf{s}_{s}^{I} ; \mathbf{y}_{d}^{I}\right)+(1-\alpha) I\left(\mathbf{s}_{r}^{I I} ; \mathbf{y}_{d}^{I I}\right)\right\},
\end{aligned}
$$

and

$$
\begin{aligned}
C_{\text {coop }}\left(\gamma_{e}^{o}, \alpha\right) \geq & \min \left\{\alpha I\left(\mathbf{s}_{s}^{I} ; \mathbf{y}_{r}^{I}\right),\right. \\
& \left.\alpha I\left(\mathbf{s}_{s}^{I} ; \mathbf{y}_{d}^{I}\right)+(1-\alpha) I\left(\mathbf{s}_{r}^{I I} ; \mathbf{y}_{d}^{I I}\right)\right\},
\end{aligned}
$$

where $I(\mathbf{a} ; \mathbf{b})$ represents the average mutual information (MI) between the channel input a and the corresponding channel output $\mathbf{b}$, while the TRA-factor $\alpha$ is defined as:

$$
\alpha \triangleq \frac{L_{s}}{L_{s}+L_{r}}=\frac{R_{r}}{R_{s}+R_{r}}
$$

since the ratio of the time durations $\left(L_{s}, L_{r}\right)$ used by the source and RS is inversely proportional to the ratio of the channel code rates $\left(R_{s}, R_{r}\right)$ employed by them ${ }^{1}$. Note that the capacity upper and lower bounds of (4) and (5) are functions of the network's overall equivalent $\mathrm{SNR}^{2}$, i.e. $\gamma_{e}^{o}$ and the TRAfactor $\alpha$ of (6). The constrained information rates of $I\left(\mathbf{s}_{s}^{I} ; \mathbf{y}_{d}^{I}\right)$, $I\left(\mathbf{s}_{r}^{I I} ; \mathbf{y}_{d}^{I I}\right), I\left(\mathbf{s}_{s}^{I} ; \mathbf{y}_{r}^{I}\right)$ and $I\left(\mathbf{s}_{s}^{I} ; \mathbf{y}_{d}^{I}, \mathbf{y}_{r}^{I}\right)$ can be evaluated using the method presented in [8].

On the other hand, given a fading block size $T_{b}$ and a $M_{c}$-ary DPSK scheme, the actual transmission rate, $R_{\text {coop }}$, of the cooperative system of Fig. 1 is a function of both $R_{s}$ and $\alpha$, which may be expressed as $R_{\text {coop }}\left(R_{s}, \alpha\right)=$ $\alpha R_{s} \frac{T_{b}-1}{T_{b}} \log _{2} M_{c}$, where the ratio of $\frac{T_{b}-1}{T_{b}}$ accounts for the modest rate-loss induced by the known reference symbol of the classic differential signalling process. Furthermore, in order for the RS to decode the received signal correctly thus avoiding the potential error propagation, the source transmission rate should be below the non-coherent constrained information rate of the source-relay link, thus we have:

$$
R_{\text {coop }}\left(R_{s}, \alpha\right) \leq \alpha C_{s r}\left(\gamma_{e}^{o}\right)=\alpha I\left(\mathbf{s}_{s}^{I} ; \mathbf{y}_{r}^{I}\right) .
$$

Consequently, according to (4), (5) and (7), the DDF-aided cooperative network capacity can be written as:

$$
\begin{aligned}
& C_{\text {coop }}^{\mathrm{DDF}}\left(\gamma_{e}^{o}, \alpha\right) \\
= & \min \left\{\alpha I\left(\mathbf{s}_{s}^{I} ; \mathbf{y}_{r}^{I}\right), \alpha I\left(\mathbf{s}_{s}^{I} ; \mathbf{y}_{d}^{I}\right)+(1-\alpha) I\left(\mathbf{s}_{r}^{I I} ; \mathbf{y}_{d}^{I I}\right)\right\},
\end{aligned}
$$

\footnotetext{
${ }^{1}$ The flexibility of code-rate-allocation may be ensured by rateless codes, for example.

${ }^{2}$ The terminology of 'equivalent SNR' is used here to indicate the fact that it quantifies the ratio of the transmit power and the receiver's noise, which are measured at physically different points. $\gamma_{e}^{o}$ denotes the network's overall equivalent SNR, having the following relationship with the equivalent SNRs, $\gamma_{e}^{s}$ and $\gamma_{e}^{r}$ at the source and relay transmitters: $\gamma_{e}^{s}+\gamma_{e}^{r}=\gamma_{e}^{o}=P / 2 \sigma_{w}^{2}$.
}

indicating that the TRA-factor $\alpha$ of (6) plays a crucial role in determining the network's capacity.

\section{B. Optimum Time Resource Allocation for the DDF System}

Lemma 1: The optimum TRA-factor $\hat{\alpha}^{o p t}$, which maximizes the achievable information rate of a single-relay-aided DDFbased cooperative transmission, is given by:

$$
\hat{\alpha}^{o p t}\left(\gamma_{e}^{o}\right)=\frac{I\left(\mathbf{s}_{r}^{I I} ; \mathbf{y}_{d}^{I I}\right)}{I\left(\mathbf{s}_{s}^{I} ; \mathbf{y}_{r}^{I}\right)-I\left(\mathbf{s}_{s}^{I} ; \mathbf{y}_{d}^{I}\right)+I\left(\mathbf{s}_{r}^{I I} ; \mathbf{y}_{d}^{I I}\right)},
$$

yielding the globally maximum achievable rate of

$$
C_{\text {coop }}^{\mathrm{DDF}}\left(\gamma_{e}^{o}, \hat{\alpha}^{o p t}\right)=\frac{I\left(\mathbf{s}_{s}^{I} ; \mathbf{y}_{r}^{I}\right) I\left(\mathbf{s}_{r}^{I I} ; \mathbf{y}_{d}^{I I}\right)}{I\left(\mathbf{s}_{s}^{I} ; \mathbf{y}_{r}^{I}\right)-I\left(\mathbf{s}_{s}^{I} ; \mathbf{y}_{d}^{I}\right)+I\left(\mathbf{s}_{r}^{I I} ; \mathbf{y}_{d}^{I I}\right)} .
$$

Proof of Lemma 1: For a given $\gamma_{e}^{o}$, we can find $\alpha=a$, which satisfies $a I\left(\mathbf{s}_{s}^{I} ; \mathbf{y}_{d}^{I}\right)+(1-a) I\left(\mathbf{s}_{r}^{I I} ; \mathbf{y}_{d}^{I I}\right)=a I\left(\mathbf{s}_{s}^{I} ; \mathbf{y}_{r}^{I}\right)$, hence the resultant network capacity of (8) becomes:

$$
\begin{aligned}
C_{\text {coop }}^{\mathrm{DDF}}\left(\gamma_{e}^{o}, a\right) & =a I\left(\mathbf{s}_{s}^{I} ; \mathbf{y}_{d}^{I}\right)+(1-a) I\left(\mathbf{s}_{r}^{I I} ; \mathbf{y}_{d}^{I I}\right) \\
& =a I\left(\mathbf{s}_{s}^{I} ; \mathbf{y}_{r}^{I}\right) .
\end{aligned}
$$

Furthermore, due to the path-loss-induced power gain, which implies that we have $\sigma_{h_{s d}}^{2}<\sigma_{h_{r d}}^{2}$, and owing to the equal power allocation assumption, i.e. $P_{s}=P_{r}$, it is evident that

$$
I\left(\mathbf{s}_{s}^{I} ; \mathbf{y}_{d}^{I}\right)<I\left(\mathbf{s}_{r}^{I I} ; \mathbf{y}_{d}^{I I}\right) .
$$

Thus, for any $\alpha=b>a$, we have:

$b I\left(\mathbf{s}_{s}^{I} ; \mathbf{y}_{d}^{I}\right)+(1-b) I\left(\mathbf{s}_{r}^{I I} ; \mathbf{y}_{d}^{I I}\right)<a I\left(\mathbf{s}_{s}^{I} ; \mathbf{y}_{d}^{I}\right)+(1-a) I\left(\mathbf{s}_{r}^{I I} ; \mathbf{y}_{d}^{I I}\right)$,

which in turn results in

$$
b I\left(\mathbf{s}_{s}^{I} ; \mathbf{y}_{d}^{I}\right)+(1-b) I\left(\mathbf{s}_{r}^{I I} ; \mathbf{y}_{d}^{I I}\right)<a I\left(\mathbf{s}_{s}^{I} ; \mathbf{y}_{r}^{I}\right)<b I\left(\mathbf{s}_{s}^{I} ; \mathbf{y}_{r}^{I}\right) .
$$

Hence, according to (8), the network capacity associated with $\alpha=b$ may be expressed as:

$$
C_{\text {coop }}^{\mathrm{DDF}}\left(\gamma_{e}^{o}, b\right)=b I\left(\mathbf{s}_{s}^{I} ; \mathbf{y}_{d}^{I}\right)+(1-b) I\left(\mathbf{s}_{r}^{I I} ; \mathbf{y}_{d}^{I I}\right) .
$$

Based on (11), (13) and (15), we arrive at:

$$
C_{\text {coop }}^{\mathrm{DDF}}\left(\gamma_{e}^{o}, b\right)<C_{\text {coop }}^{\mathrm{DDF}}\left(\gamma_{e}^{o}, a\right), \quad \text { if } b>a \text {. }
$$

On the other hand, according to (11) and (12), for any $\alpha=$ $c<a$, we have:

$$
\begin{aligned}
& c I\left(\mathbf{s}_{s}^{I} ; \mathbf{y}_{d}^{I}\right)+(1-c) I\left(\mathbf{s}_{r}^{I I} ; \mathbf{y}_{d}^{I I}\right) \\
> & a I\left(\mathbf{s}_{s}^{I} ; \mathbf{y}_{d}^{I}\right)+(1-a) I\left(\mathbf{s}_{r}^{I I} ; \mathbf{y}_{d}^{I I}\right)=a I\left(\mathbf{s}_{s}^{I} ; \mathbf{y}_{r}^{I}\right) .
\end{aligned}
$$

Additionally, since $c<a$, we have:

$$
c I\left(\mathbf{s}_{s}^{I} ; \mathbf{y}_{r}^{I}\right)<a I\left(\mathbf{s}_{s}^{I} ; \mathbf{y}_{r}^{I}\right),
$$

which in turn yields $c I\left(\mathbf{s}_{s}^{I} ; \mathbf{y}_{d}^{I}\right)+(1-c) I\left(\mathbf{s}_{r}^{I I} ; \mathbf{y}_{d}^{I I}\right)>$ $c I\left(\mathbf{s}_{s}^{I} ; \mathbf{y}_{r}^{I}\right)$. Again, according to (8), the maximum achievable information rate associated with $\alpha=c$ may be formulated as:

$$
C_{\text {coop }}^{\mathrm{DDF}}\left(\gamma_{e}^{o}, c\right)=c I\left(\mathbf{s}_{s}^{I} ; \mathbf{y}_{r}^{I}\right) \text {. }
$$

Consequently, by referring to (11), (18) and (19), it becomes plausible that:

$$
C_{\text {coop }}^{\mathrm{DDF}}\left(\gamma_{e}^{o}, c\right)<C_{\text {coop }}^{\mathrm{DDF}}\left(\gamma_{e}^{o}, a\right), \quad \text { if } c<a .
$$




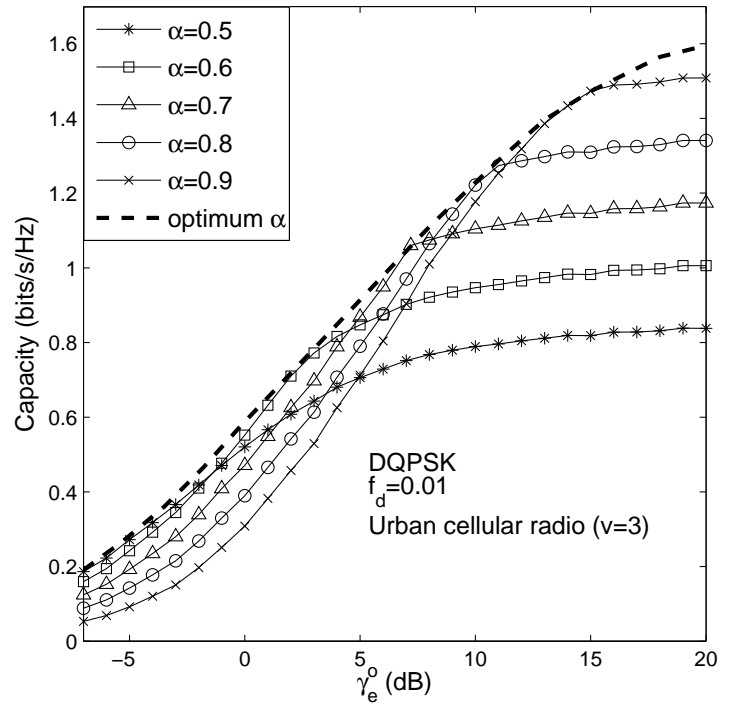

Fig. 2. Capacity of the single-relay-aided DDF cooperative system.

Hence, based on (16) and (20), the optimum TRA-factor that maximizes the achievable network information rate is $\alpha^{\text {opt }}=$ $a$, which can be computed with the aid of (11). Fianlly, we arrive at:

$$
\hat{\alpha}^{o p t}\left(\gamma_{e}^{o}\right)=\frac{I\left(\mathbf{s}_{r}^{I I} ; \mathbf{y}_{d}^{I I}\right)}{I\left(\mathbf{s}_{s}^{I} ; \mathbf{y}_{r}^{I}\right)-I\left(\mathbf{s}_{s}^{I} ; \mathbf{y}_{d}^{I}\right)+I\left(\mathbf{s}_{r}^{I I} ; \mathbf{y}_{d}^{I I}\right)},
$$

which in turn leads to the globally maximum achievable network information rate as:

$$
C_{\text {coop }}^{\mathrm{DDF}}\left(\gamma_{e}^{o}, \hat{\alpha}^{\text {opt }}\right)=\frac{I\left(\mathbf{s}_{s}^{I} ; \mathbf{y}_{r}^{I}\right) I\left(\mathbf{s}_{r}^{I I} ; \mathbf{y}_{d}^{I I}\right)}{I\left(\mathbf{s}_{s}^{I} ; \mathbf{y}_{r}^{I}\right)-I\left(\mathbf{s}_{s}^{I} ; \mathbf{y}_{d}^{I}\right)+I\left(\mathbf{s}_{r}^{I I} ; \mathbf{y}_{d}^{I I}\right)}
$$

This completes the proof of Lemma 1.

\section{NumericAl EVAluation AND Discussions}

In Fig. 2 the single-relay-aided cooperative system's capacity associated with different values of $\alpha$ is depicted versus $\gamma_{e}^{o}$ using (8) in comparison to that of its adaptive-TRA-aided counterpart in conjunction with the optimum $\alpha$ of (9) in a typical urban cellular radio scenario associated with the path loss exponent of $v=3$. It is observed that the latter exhibits significant capacity gains over the former with the aid of the adaptive TRA scheme.

In order to gain further insights into the benefits of the single-relay-assisted DDF cooperative system over its conventional direct-transmission based counterpart from a pure capacity perspective, the capacity of the adaptive-TRA-aided cooperative system is depicted in comparison to that of the direct-transmission based one in Fig. 3. It may be observed in the figure that when the overall equivalent SNR is relatively low, the TRA-optimized DDF cooperative system exhibits a significantly higher capacity than its direct-transmission based counterpart in typical urban cellular radio scenarios. More specifically, in a shadowed urban area associated with $v=4$, the DDF system only requires one third of the total transmit power necessitated by its direct-transmission based counterpart

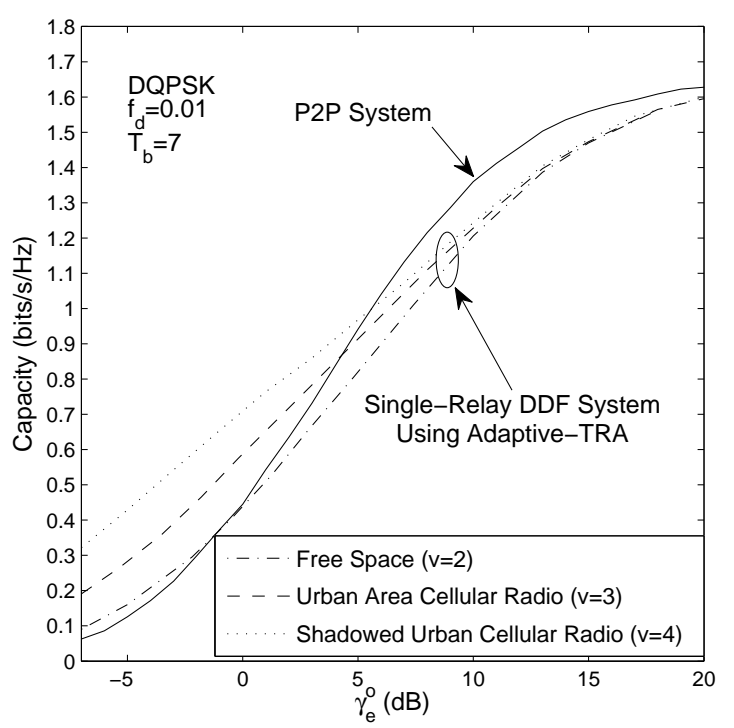

Fig. 3. Capacity comparison of the single-relay-aided cooperative system and its direct-transmission based counterpart.

in order to achieve a spectral efficiency of $0.5 \mathrm{bits} / \mathrm{s} / \mathrm{Hz}$. However, the achievable capacity gain may be substantially reduced, if we encounter a free-space propagation scenario associated with $v=2$, since the reduced-path-loss-related power-gain achieved is insufficiently high to compensate for the significant multiplexing loss inherent in the single-relayaided half-duplex TDMA system. Moreover, as the overall equivalent SNR increases to a relatively high value, the benefits of invoking a single-relay-aided cooperative system for achieving a high spectral efficiency erode.

\section{CONCLUSION}

In this letter, we proposed an optimum TRA scheme for the DDF cooperative system, which was verified to be capable of maximizing the network capacity through the analysis and numerical simulations.

\section{REFERENCES}

[1] J. N. Laneman, D. N. C. Tse, and G. W. Wornell, "Cooperative diversity in wireless networks: Efficient protocols and outage behavior," IEEE Transaction on Information Theory, vol. 50, pp. 3062-3080, Dec. 2004.

[2] K. G. Seddik, A. K. Sadek, W. Su, and K. J. R. Liu, "Outage analysis and optimal power allocation for multinode relay networks," IEEE Signal Processing Letters, vol. 14, pp. 377-380, June 2007.

[3] T. Himsoon, W. Su, and K. J. R. Liu, "Differential transmission for amplify-and-forward cooperative communications," IEEE Signal Processing Letters, vol. 12, pp. 597-600, Sept. 2005.

[4] Q. Zhao and H. Li, "Performance of differential modulation with wireless relays in rayleigh fading channels," IEEE Communications Letters, vol. 9, pp. 343-345, Apr. 2005.

[5] L. Wang and L. Hanzo, "The amplify-and-forward cooperative uplink using multiple-symbol differential sphere-detection," IEEE Signal Processing Letters, vol. 16, pp. 913-916, Oct. 2009.

[6] L. Wang and L. Hanzo, "The resource-optimized differentially modulated hybrid $\mathrm{AF} / \mathrm{DF}$ cooperative cellular uplink using multiple-symbol differential sphere detection," IEEE Signal Processing Letters, vol. 16, pp. 965-968, Nov. 2009.

[7] A. Hst-Madsen and J. Zhang, "Capacity bounds and power allocation for wireless relay channel," IEEE Transactions on Information Theory, vol. 51, pp. 2020-2040, June 2005.

[8] R.-R. Chen, R. Koetter, and U. Madhow, "Joint noncoherent demodulation and decoding for fast Rayleigh fading channels," Conference on Information Sciences and Systems, Mar. 2003. 